\title{
Osteotomien nach in Achsenabweichung verheilten Frakturen des Handskeletts
}

\author{
R. Friedel, E. Markgraf, T. Dönicke, S. Mohr
}

\section{Zusammenfassung}

Die Versorgung von knöchernen Verletzungen des Handskeletts hat sich in den letzten Jahren durch die rasante Entwicklung von neuen Osteosynthesetechniken mehr zur operativen Therapie gewandelt. Die Entwicklung von Mikroplatten und Schrauben ermöglicht zunehmend die Stabilisierung von komplizierten gelenknahen Frakturen. Massengill u. Mitarb. [3] sowie Black u. Mitarb. [1] konnten anhand von biomechanischen Untersuchungen zeigen, dass die höchste Stabilität durch Platten und Schrauben erreicht wird. Trotzdem muss insbesondere bei komplexen Handverletzungen gelegentlich noch auf K-Drahtosteosynthesen zurückgegriffen werden. Nach unseren Erfahrungen besteht hier besonders die Gefahr von Achs- und Rotationsfehlstellungen der Finger- und Mittelhandknochen (Abb.1). Auch nach unzureichender Immobilisation bei konservativer Frakturbehandlung werden häufig Achs- und Rotationsfehlstellungen beobachtet [7]. Diese werden vom Patienten nicht toleriert, weil sie zur Behinderung des Faustschlusses sowie durch Überkreuzen der Finger zur Störung der Greiffunktion führen. Defektfrakturen können zusätzlich noch Längendifferenzen bedingen, welche das komplizierte Sehnenspiel erheblich beeinträchtigen. Achsfehlstellungen können primär nach fehlerhaften Osteosynthesen, insbesondere bei Trümmerfrakturen, und sekundär durch Fehlheilung (Pseudarthrosen, Infekt) auftreten. Der Zeitpunkt der Korrektur hängt vom Frakturtyp und den Begleitverletzungen ab. Bei Trümmerfrakturen erfolgt die Korrekturosteotomie nach der Frakturkonsolidierung, wohingegen bei einfachen Frakturen die Korrektur noch vor der Ausheilung durchzuführen ist. Bei den meisten Patienten liegen Kombinationsfehlstellungen vor. Deshalb ist eine genaue präoperative Analyse der Fehlstellung und Planung der Osteosynthese erforderlich. Die Korrekturebene richtet sich nach der Art und Höhe der Fehlstellung. Das Ziel der Korrektur ist die dreidimensionale Wiederherstellung der ursprünglichen Skelettachse und -Länge. Als Implantate für die übungsstabilen Osteosynthesen kommen bevorzugt Platten und Schrauben in Betracht.

\section{Einleitung}

Korrekturosteotomien nach in Fehlstellung ausgeheilten Frakturen der Mittelhand- und Fingerknochen sind seltene Operationen. In unserem Krankengut waren bei 3025 handchirurgischen Eingriffen in den letzten 10 Jahren 27 Korrektur-

OP-JOURNAL 2000; 16: 40-43

(c) Georg Thieme Verlag Stuttgart · New York osteotomien (0,9\%) erforderlich. In der Literatur werden nur Ergebnisse über geringe Fallzahlen vorgelegt $[4,8,6]$. Deshalb sollten diese Eingriffe von erfahrenen Handchirurgen durchgeführt werden. Eine präoperative Skizze mit Bestimmung der Korrekturwinkel hat sich als sinnvoll erwiesen.

Man findet prinzipiell Achsfehlstellungen in der Handebene, nach dorsal und palmar, sowie Rotationsfehler. Die Notwendigkeit der Korrektur ergibt sich aus dem funktionellen Defizit sowie den speziellen Wünschen des Patienten.
Die Zugänge entsprechen den allgemeinen Regeln der Handchirurgie und sind von dorsal zu planen. Bei erheblicher Narbenbildung nach komplexen Handverletzungen müssen die vorhandenen Narben sinnvoll in den Zugang einbezogen werden, um Hautnekrosen mit sekundärer Infektion zu vermeiden. Besonders gravierend sind ulnare Deviationen des Zeigefingers, die sowohl den Spitzgriff als auch den Faustschluss stören. Die Osteosynthese nach Korrektur sollte eine sofortige funktionelle Beübung erlauben. Die operative Planung und Nachbehandlung muss mit dem Patienten entsprechend seiner Bedürfnisse und Wünsche detailiert abgesprochen werden. Dabei hat es sich als nützlich erwiesen, dem Patienten die operative Vorgehensweise und Nachbehandlung an ähnlich gelagerten klinischen Fällen zu demonstrieren (Skizzen, Dias, Videos).

\section{Endglieder}

Im Bereich der Endglieder sind Drehfehler meist ohne funktionelle Bedeutung. Achsenfehlstellungen im Endgliedbereich können den Patienten aber behindern. Hier ist in aller Regel die Endgliedarthrodese mit Achskorrektur indiziert. Zur Vermeidung einer Verkürzung kann die Arthrodese mit einer Spongiosaplastik vom Radius kombiniert werden. Wir bevorzugen orthograde Schraubenarthrodesen mit $1,5 \mathrm{~mm}$ Titan-Schrauben.

Fehlstellungen der Endglieder sind selten korrekturbedürftig

\section{Mittelglieder}

Im Bereich der Mittelphalangen erfolgt die Korrektur von Achsfehlstellungen nach radial und ulnar am Ort der Fehlstellung mittels Keilosteotomie. Achsfehlstellungen nach dorsal und palmar behindern im Wesentlichen die Fingerbeugung und Streckung. Hier ist in seltenen Fällen nach Absprache mit dem Patienten eine 

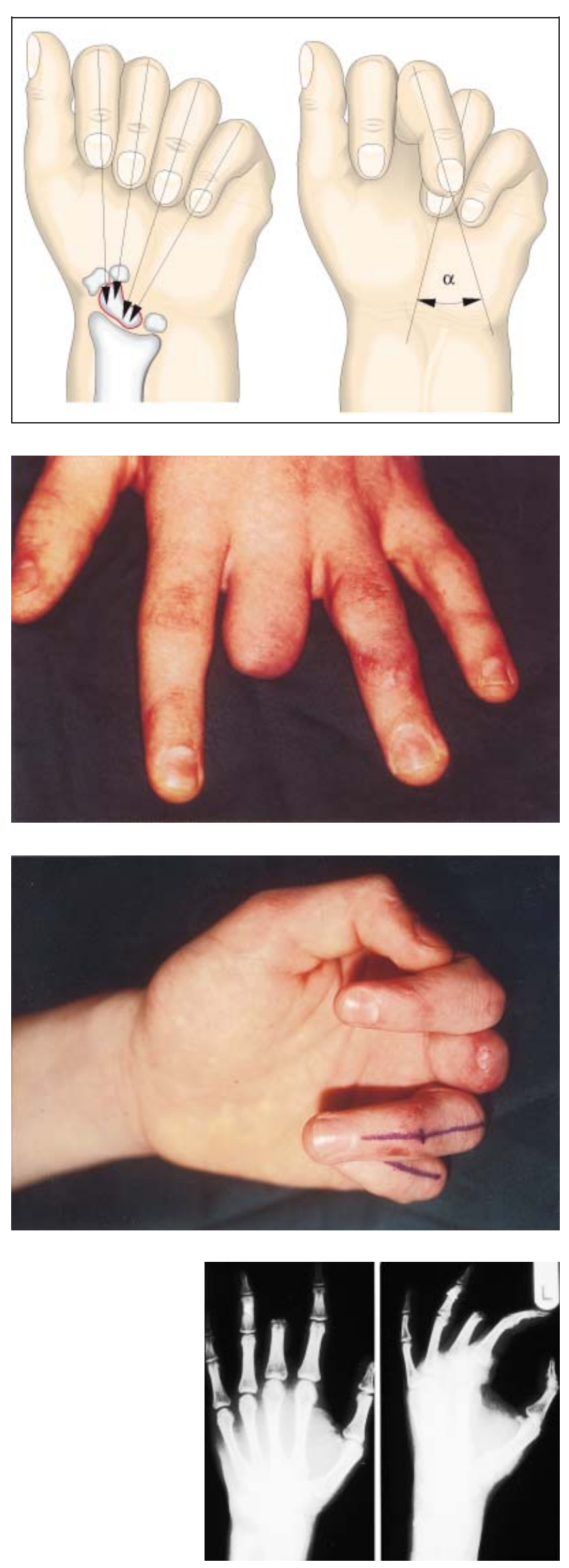

Abb. 1 Die Achsen der Langfinger konvergieren bei Beugung auf die Tuberositas des Os scaphoideum. Bereits kleine Rotationsfehler der Phalangen bedingen ein Überschneiden der Finger mit erheblichen funktionellen Problemen für den $\mathrm{Pa}$ tienten.

Abb. 2a Patient, 19 Jahre, männl., 20 Grad Rotationsfehlstellung 4. Finger links nach komplexer Kreissägenverletzung vor 2 Monaten.

Abb. 2 b

Der 4. Finger überkreuzt bei Beugung den 5. Finger.

Abb. 2c Primäre Osteosynthese der Mittelphalanxfraktur mit 2 Zugschrauben.
Keilosteotomie indiziert. Eine dorsale Abweichung im Grundglied kann zur Insuffizienz des Mittelzügels und zu Abweichungen der Seitenzügel führen. Bei der Osteosynthese sollten Verwachsungen zwischen Strecksehne und Osteotomiestelle durch seitliche Osteosynthesen mit Kondylenplatten vermieden werden. Bei der Verwendung von dorsalen Miniplatten muss das Periost über der Platte subtil verschlossen werden. Rotationsfehler der Mittelphalangen stören bei gestreckten Fingern nur wenig. Beim Faustschluss kommt es jedoch zur Überschneidung der Fingerachsen mit erheblichen funktionellen Problemen. Hier ist in der Regel die technisch einfache subkapitale, quere Osteotomie der Mittelhandknochen durchzuführen.

Bei den meisten Patienten handelt es sich um Kombinationsfehlstellungen. Dabei kommt es je nach überwiegender Komponente zu Problemen beim Faustschluss oder der Fingerbeugung/Streckung. Nach genauer präoperativer Analyse sollte die Korrektur am Ort der Fehlstellung in der Weise durchgeführt werden, dass die Hauptkomponente nach Ausmessen des Fehlers in Winkelgraden unter Berücksichtigung der Nebenkomponente korrigiert wird. Als Osteosynthesematerial haben sich Titan-Platten und -schrauben der Dimension 1,5 mm bewährt. Der ausgemessene Korrekturwinkel wird vor und nach der Osteotomie durch temporäre K-Drähte markiert (Abb.3). Noch vor definitiver Fixation der Plattenosteosynthese wird intraoperativ durch passiven Faustschluss die Korrektur überprüft. In dieser Stellung erfolgt die Entfernung der KDrähte und die definitive Fixation der Plattenosteosynthese (Abb.4). Achsfehlstellungen der Finger können auch infolge von postraumatischen, postinfektiösen oder chronisch-entzündlichen Gelenkdeformitäten entstehen. In ausgewählten Fällen bieten sich dabei Korrekturoperationen im Gelenkbereich an, die neben dem Ausgleich der Achsfehlstellung auch einen Erhalt der Gelenkfunktion zum Ziel haben sollten. Beispielsweise kann dazu eine unikondyläre Korrekturosteotomie Anwendung finden [5].

Je näher die Fehlstellung am PIP-Gelenk ist, um so ausgeprägter ist die funktionelle Einschränkung.

\section{Grundglieder}

Fehlstellungen nach Grundgliedfrakturen können von dorsal am Ort der Fehlstellung oder am Metakarpaleköpfchen kor- 
rigiert werden. Die Umstellung am Mittelhandköpfchen hat den Vorteil, dass der Zugang das Gleiten der Strecksehnen weniger tangiert und die Operation technisch einfacher ist [2]. Klinisch tritt der Nachteil der Korrektur über das MCP-Gelenk nicht wesentlich in Erscheinung.

Eine Alternative bei der Korrektur von Rotationsfehlern der Grundglieder und Metacarpalia zur queren Osteotomie ist die Z-förmige Osteotomie.

Dabei wird die Z-förmige Osteotomie bei nach ulnar gerichteter Fehlrotation der Fingerkuppe distal nach ulnar und bei nach radial gerichteten Rotationsfehlern distal nach radial positioniert. Zwei quere Kompressionsschrauben reichen dann für die übungsstabile Osteosynthese aus. Bei dieser Methode wird allerdings der Vorteil der höheren Stabilität durch eine aufwendigere Operationstechnik neutralisiert.

Kombinationsfehlstellungen sind immer am Ort der Fehlstellung zu korrigieren.

Als Osteosynthesematerial stehen Kondylenplatten, T-Platten und K-Drähte zur Verfügung. In seltenen Fällen von Rotationsfehlstellungen, insbesbesondere am Zeigefingergrundglied, und zusätzlichen osteitischen Befunden ist die Zeigefingeramputation nach Adelmann zur Verbesserung der Funktion indiziert.

Grundsätzlich sollte vor der Korrektur einer Fehlstellung die Sensibilität des Fingers wiederhergestellt werden.

\section{Mittelhandknochen}

Rotationsfehler im Grundgliedbereich werden durch Korrektur subkapital am Os metacarpale ausgeglichen. Bereits bei der Planung der Korrekturosteotomie muß man berücksichtigen, dass die Achsen der Finger bei der Beugung auf die Tuberositas des Os scaphoideum konvergieren (Abb.1).

Nach Frakturen der Ossa metacarpalia treten bei intakten interossären Ligamenten selten Rotationsfehler auf.

Prinzipiell besteht im Falle eines Rotationsfehlers eines Mittelhandknochens auch die Option der Z-förmigen Keilosteotomie.

Häufiger sind Verkürzungen nach Defektoder Trümmerfrakturen, welche das natürliche Alignement der MCP-Gelenke und das Sehnengleichgewicht stören.

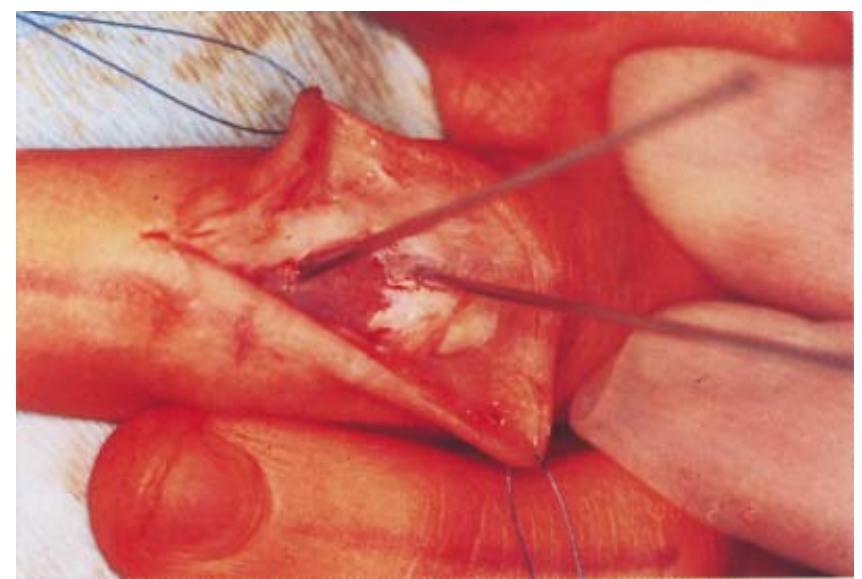

Abb. 3 Rotationskorrektur an der Basis des Grundgliedes um 20 Grad. Temporäres Einbringen von K-Stiften distal und proximal der vorgesehenen Osteotomie zur Darstellung des Korrekturwinkels von 20 Grad.

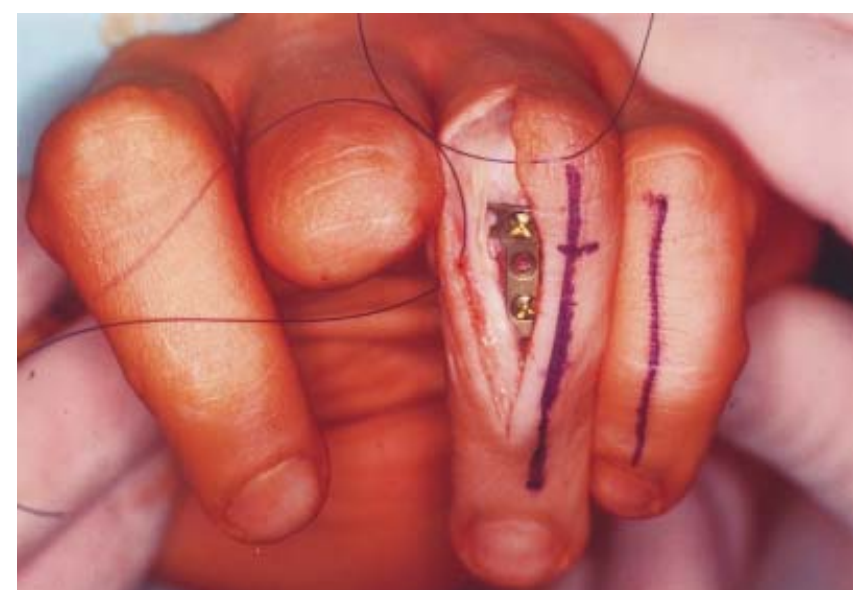

Abb.4 Definitive Fixation der Platte nach Osteotomie in Beugestellung des Fingers.

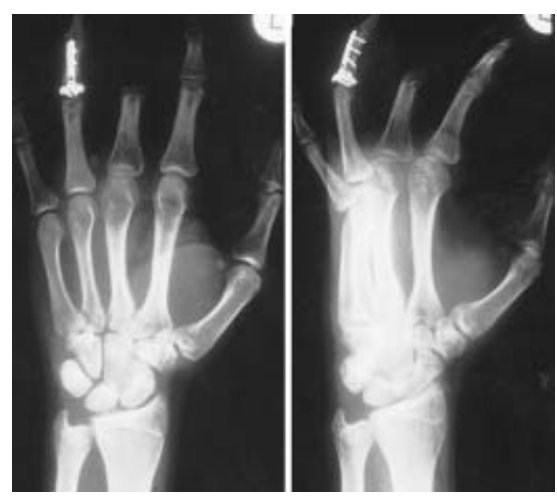

Abb. 5a Postoperativer Röntgenbefund.

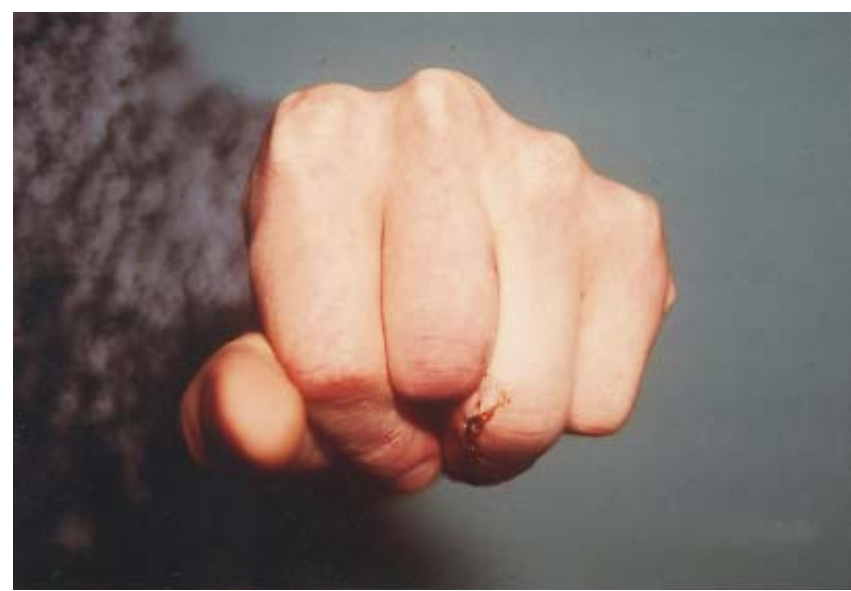

Abb. 5b Postoperatives funktionelles Ergebnis nach 3 Wochen. Der Faustschluss ist wieder normal möglich. 
Die Längenkorrektur kann über Kallusdistraktion oder durch eine einzeitige Interposition eines kortiko-spongiösen Spanes erfolgen. Als Osteosynthesematerial kommen Titan-Platten der Dimension 2,0 mm sowie der Fixateur externe zur Anwendung.

\section{Fallbeschreibung}

Nach Versorgung einer komplexen Handverletzung bei einem 19-jährigen Patienten kam es am 4. Finger zu einer ulnaren Rotationsfehlstellung von 20 Grad im Mittelphalanxbereich (Abb.2). Ursache hierfür war eine fehlerhafte primäre Zugschraubenosteosynthese. Die dadurch auftretende Überschneidung des 4. Fingers auf den 5. Finger war für den Patienten funktionell besonders nachteilig, da der Mittelfinger bei der primären Versorgung im distalen Drittel der Mittelphalanx amputiert werden musste und somit eine mediane Lücke beim Grobgriff resultierte. Nach Entfernung der Minischrauben wurde eine quere Osteotomie der Mittelphalanxbasis angelegt. Mit temporären K-Stiften läßt sich der Rotationsfehler gut darstellen (Abb.3). Vor der definitiven Fixation der Miniplatte wird durch passive Beugung der Finger die korrekte Achse überprüft (Abb.4). Das funktionelle Ergebnis war für den Patienten befriedigend (Abb.5).

\section{Schlussfolgerungen}

Prinzipiell sollten bei jeder primären Osteosynthese Rotations-, Achs- und Längenfehler vermieden werden. Inadäquat konservativ behandelte Handfrakturen führen nicht selten zur Ausheilung in Fehlstellung. Funktionell machen sich besonders Rotations- und Achsenfehlstellungen der Grund- und Mittelphalangen in Gelenknähe negativ bemerkbar. Eine genaue präoperative Analyse und Dokumentation der Fehlstellung und Planung des operativen Vorgehens ist unbedingt erforderlich. Stabile Plattenosteosynthesen mit der Möglichkeit der sofortigen Übungsbehandlung sind instabilen KDrahtosteosynthesen vorzuziehen. Das Ziel der Korrekturosteotomie ist die dreidimensionale Wiederherstellung der ursprünglichen Anatomie und Funktion des Handskeletts.

\section{Literatur}

1 Black D, Mann, RJ, Constine R, Daniels AU. Comparison of Internal Fixation Techniques in metacarpal Fractures. J Hand Surg 1985; 10a: 466-472

2 Lopatecki M, Rudigier J. Korrekturosteotomien an der Hand OP-Journal 1991; 2 . $68-72$

3 Massengill JB, Alexander H, Langrana N, Mylod A. A. phalangeal fracture Model - Quantitative Analysis of Rigidity and Failure. J Hand Surg 1982; 7: 264-270
4 Menon J. Correction of rotary malunion of the fingers by metacarpal rotational osteotomy. Orthopedics 1990 Febr; 13(2): 197-200

5 Obiltschnig A, Mauhart G. Korrektur der Fehlstellung eines Ringfingers nach Kirschner-Draht-Infektion des Mittelgelenkes. Ein Fallbericht. Handchir Mikrochir Plast Chir 1999; 31: 212 -214

${ }^{6}$ Pichora DR, Meyer R, Masear VR. Rotational step-cut osteotomy for treatment metacarpal phalangeal malunion. J Hand Surg [Am] 1991 May; 16(3): 551-5

7 Renner A, Santha E, Manninger J. Corrective osteotomy in malalinment of healed fractures metacarpal and phalangeal bones. Handchirurgie 1979; 11(2-4): 213-8

8 Trumble T, Gilbert M. In situ osteotomy for extra-articular malunion of the proximal phalanx. J Hand Surg [Am] 1998 Sept; 23(5): $821-6$

\section{Dr. med. R. Friedel}

Oberarzt der Klinik für Unfallchirurgie Leiter Arbeitsbereich Handchirurgie

\section{T. Dönicke}

Assistenzarzt Arbeitsbereich

Handchirurgie

\section{S. Mohr}

AIP Arbeitsbereich Handchirurgie

Prof. Dr. med. habil. E. Markgraf Direktor der Klinik für Unfallchirurgie

Klinikum der Friedrich-Schiller-

Universität Jena

Klinik für Unfallchirurgie

Bachstr. 18

07740 Jena

Fax: 03641/933978

E-Mail: Reinhard Friedel@med.unijena.de 